\title{
Crescimento de Aegla longirostri Bond-Buckup \& Buckup (Crustacea, Anomura, Aeglidae)
}

\author{
Daiana da Silva-Castiglioni ${ }^{1}$, Daniela F. Barcelos ${ }^{1} \&$ Sandro Santos ${ }^{2}$
}

\author{
1 Programa de Pós-graduaçãm em Biologia Animal, Departamento de Zoologia, Universidade Federal do Rio Grande do Sul. \\ Avenida Bento Gonçalves 9500, 91501-970 Porto Alegre, Rio Grande do Sul, Brasil. \\ ${ }^{2}$ Departamento de Biologia, Universidade Federal de Santa Maria. Faixa de Camobi, Km 09, 97105-900 Santa Maria, Rio \\ Grande do Sul, Brasil. E-mail: ssantos@smail.ufsm.br
}

\begin{abstract}
Growth of Aegla longirostri Bond-Buckup \& Buckup (Crustacea, Anomura, Aeglidae). The growth of Aegla longirostri Bond-Buckup \& Buckup, 1994 was carried out from monthly samples at Rio Ibicuí-Mirim, Itaára, locality of Val-de-Serra, State of Rio Grande do Sul, Brazil, from September 1996 to August 1997. The sex and the cephalothorax length were recorded for each captured specimens. The growth study was estimated using the von Bertalanffy's model. The growth curves in cephalothorax length ( $\mathrm{mm})$ for males and females are described, respectively, by the equations: $L t=27.9\left[1-\mathrm{e}^{-0.0069(t+24.78)}\right]$ and $L t=21.45\left[1-\mathrm{e}^{-0.0048(t+45.62)}\right]$. The maximum estimated age for males was one year and nine months and for females, two years.

KEY WORDS. Decapoda; frequency distribution; growth; von Bertalanffy.
\end{abstract}

RESUMO. O crescimento de Aegla longirostri Bond-Buckup \& Buckup, 1994 foi analisado mensalmente de setembro de 1996 a agosto de 1997 no Rio Ibicuí-Mirim, Itaára, localidade de Val-de-Serra, Estado do Rio Grande do Sul, Brasil. O sexo dos espécimes coletados foi registrado e a medida do comprimento do cefalotórax foi tomada com um paquímetro. O crescimento foi estimado usando o modelo de von Bertalanffy. As curvas de crescimento em comprimento do cefalotórax $(\mathrm{mm})$ de machos e fêmeas foram descritas, respectivamente, pelas equações: $\mathrm{Ct}$ $=27,9\left[1-\mathrm{e}^{-0,0069(\mathrm{t}+24,78)}\right]$ e $\mathrm{Ct}=21,45\left[1-\mathrm{e}^{-0,0048(\mathrm{t}+45,62)}\right]$. A idade máxima estimada para machos foi de um ano e nove meses e para fêmeas dois anos.

PALAVRAS-CHAVE. Crescimento; Decapoda; distribuição de freqüência; von Betalanffy.

O crescimento é o aumento mensurável de um sistema orgânico, em peso ou comprimento, resultante da assimilação de nutrientes provenientes do ambiente. Resulta de um balanço entre os processos de anabolismo e catabolismo que ocorrem no indivíduo, sendo dependente de fatores externos (BerTALANFfy 1938).

Segundo Hartnoll (1983), as estratégias de crescimento em crustáceos são basicamente duas, o crescimento indeterminado e o determinado. Nas espécies de crescimento indeterminado, que continuam sofrendo mudas indefinidamente, a percentagem de incremento por muda declina e o período de intermuda aumenta com o passar do tempo, sendo que as taxas de incremento, muda após muda, é que vão determinar o tamanho final do animal. Nas espécies de crescimento determinado as mudas cessam quando os animais atingem a maturidade sexual, sendo que a muda terminal é coincidente com esta.

Tanto para aplicações práticas como teóricas, o crescimento deve ser representado por curvas ou modelos que o descreva de forma geral (Bagenal 1978, Pauly 1980). A curva de crescimento em uma população é a relação entre uma medida do comprimento (ou peso) e a idade dos indivíduos, sendo que a determinação da curva é de fundamental importância, pois pode fornecer informações referentes ao tamanho máximo atingido pelos animais, a idade em que este tamanho é atingido e a taxa de crescimento (SANTOS 1978, VALENTI et al. 1987).

Entre os crustáceos há um bom número de informações sobre o crescimento dos camarões continentais, destacando-se os trabalhos com Macrobrachium acanthurus (Wiegmann, 1836), Macrobrachium rosenbergii (de Man, 1879), Macrobrachium carcinus (Linnaeus, 1758), Macrobrachium potiuna (Müller, 1880), Potimirim glabra (Kingsley 1878), Exopalaemon modestus (Heller, 1862) e Palaemon longirostris H. Milne Edwards, 1837 (VAlEntr et al. 1987, 1993, 1994, Souza \& FonToura 1995, Barros \& Fontoura 1996, Он et al. 2002, CARTAXANA 2003, respectivamente).

Com relação a outros Decapoda, destacam-se os trabalhos sobre crescimento realizados por FONTOURA \& BUCKUP (1989), que analisaram o crescimento do lagostim Parastacus brasiliensis (von Martens, 1869), Branco \& Masunari (1992) com Callinectes 
danae Smith, 1869, D'INCAO et al. (1993) com Chasmagnathus granulata Dana, 1851, Cracco \& FonTOURA (1996) com Cyrtograpsus angulatus Dana, 1851 e Castiglione et al. (2004), com Uca rapax (Smith, 1870).

Em aeglídeos, o crescimento foi estudado pioneiramente por BAHAMONDE \& LOPEZ (1961), que investigaram uma população de Aegla laevis laevis Latreille, 1818 proveniente do Chile. A seguir Bueno et al. (2000) analisaram o crescimento de Aegla platensis Schmitt, 1942 no Rio Grande do Sul e Noro \& Buckup (2003) Aegla leptodactyla Buckup \& Rossi, 1977 no planalto riograndense, sendo esses dois últimos trabalhos baseados no modelo de BERTALANFFy (1938).

O objetivo deste trabalho é caracterizar o crescimento de Aegla longirostri, visando contribuir para o conhecimento de sua biologia e fornecer informações adicionais para a preservação dos aeglídeos, cuja distribuição é restrita à América do Sul e estão ameaçados de extinção pela degradação de seu ambiente natural. Esta espécie distribui-se pelo nordeste, centro e leste do Estado do Rio Grande do Sul (BOND-BUCKUP \& BucKup 1994).

\section{MATERIAL E MÉTODOS}

Os aeglídeos foram coletados mensalmente no Rio IbicuíMirim, localidade de Val-de-Serra, município de Itaára, Rio Grande do Sul, Brasil, no período de setembro de 1996 a agosto de 1997.

Os aeglídeos foram amostrados com uma rede cônica de malha de 0,5 cm entre nós, com as seguintes dimensões: 1,20 $\mathrm{m}$ de altura, $4 \mathrm{~m}$ de comprimento e 1,5 $\mathrm{m}$ de fundo. No local de coleta, levantaram-se as pedras mexendo no substrato e na vegetação de uma margem a outra do arroio, durante 15 minutos, numa área de cerca de $8 \mathrm{~m}^{2}$, sempre no sentido oposto da correnteza, para que tantos juvenis e adultos fossem levados para dentro do equipamento de captura. Os crustáceos foram colocados em sacos plásticos, fixados em álcool, para posterior triagem e identificação.

Os animais capturados foram levados para o laboratório de Carcinologia do Departamento de Biologia (Centro de Ciências Naturais e Exatas, Universidade Federal de Santa Maria) sendo identificados conforme Bond-Buckup \& Buckup (1994), separados quanto ao sexo, pela observação da presença de quatro pares de pleópodos no abdome e poro genital na coxa do terceiro par de pereiópodos em fêmeas (ausência em machos).

O comprimento do cefalotórax (CC), medido da margem interna da órbita ocular até a extremidade posterior da área cardíaca, foi tomado utilizando-se um paquímetro digital (More \& Wright), com precisão de $0,1 \mathrm{~mm}$.

O crescimento em comprimento de machos e fêmeas de A. longirostri foi analisado a partir da distribuição de freqüências absolutas em intervalos de classes de $1 \mathrm{~mm}$ de CC, que corresponde à quarta parte do desvio padrão da média calculada para o total da amostra examinada (MARkus 1971). Os indivíduos que não foram identificados quanto ao sexo, pela ausência de caracteres sexuais secundários (juvenis sem dimor- fismo sexual aparente), foram somados aos conjuntos de ambos os sexos na análise de distribuição de freqüência em classes de tamanho.

As modas dos histogramas de freqüências do comprimento do cefalotórax foram calculadas segundo SPIEgel (1979): Moda $=\mathrm{L} 1+(\Delta 1 / \Delta 1+\Delta 2) \times \mathrm{C}$, sendo: $\mathrm{L} 1=$ limite real inferior da classe modal (a que contém a moda); $\Delta 1=$ excesso da freqüência modal sobre a da classe imediatamente inferior; $\Delta 2=$ excesso da freqüência modal sobre a da classe imediatamente superior; $\mathrm{C}=$ amplitude do intervalo da classe modal.

Os cálculos para a curva de crescimento foram realizados pelo modo de agrupamento, sendo o verão considerado a primeira estação de coleta, representado pelos meses de dezembro, janeiro e fevereiro.

O modelo de crescimento utilizado para a determinação da curva de crescimento, para ambos os sexos, foi o de BERTALANFFy (1938), que corresponde à seguinte equação matemática: $\mathrm{Ct}=\mathrm{C}_{\infty}\left[1-\mathrm{e}^{-\mathrm{k}(\mathrm{t}+\mathrm{to})}\right]$, sendo $(\mathrm{Ct})$ comprimento do cefalotórax na idade $t$, em milímetros; $\left(\mathrm{C}_{\infty}\right)$ o comprimento médio máximo do cefalotórax, em milímetros; (e) base dos logaritmos neperianos; $(\mathrm{k})$ constante relacionada à taxa de crescimento; $(\mathrm{t})$ idade dos indivíduos, em dias e $\left(\mathrm{t}_{0}\right)$ parâmetro relacionado com o comprimento do animal ao nascer.

As curvas de crescimento foram linearizadas através da técnica proposta por AlLEN (1976) e as retas obtidas foram comparadas pela análise de covariância, para um intervalo de confiança de 95\%, segundo SNEDECOR \& Cochran (1967).

\section{RESULTADOS E DISCUSSÃO}

Foram mensurados 213 machos, 189 fêmeas e 202 juvenis de $A$. longirostri. O menor aeglídeo capturado apresentou 1,9 mm de comprimento do cefalotórax (juvenil com sexo indeterminado) e o maior 23,8 $\mathrm{mm}$ (macho).

A figura 1 apresenta as distribuições de freqüência sazonais do comprimento do cefalotórax $(\mathrm{mm})$ para machos e fêmeas. A maior freqüência de juvenis foi observada no período de dezembro de 1996 a fevereiro de 1997 (verão). Portanto, optou-se por acompanhar o crescimento dos animais a partir do verão e, decidiu-se incorporar ao estudo do deslocamento modal, a coorte de juvenis amostrados a partir desta estação (dezembro de 1996 a fevereiro de 1997), antepondo-se ao grupo etário representado pelos animais maiores, amostrados na primavera (setembro a novembro de 1996). Esta medida é adequada em função do crescimento de populações locais não sofrer mudanças importantes de um ano para outro (Noro \& BuCKUP 2003).

Durante as amostragens foi possível observar apenas um período de recrutamento no ano, como observado em A. laevis laevis, Aegla paulensis (Schmitt, 1942), Aegla perobae Hebling \& Rodrigues, 1977, A. platensis e A. Leptodactyla por, respectivamente, BAHAMONde \& Lopez (1961), Lopez (1965), Rodrigues \& Hebling (1978), Bueno et al. (2000) e Noro \& Buckup (2003). Somente Swiech-Ayoub \& Masunari (2001a) verificaram dois 

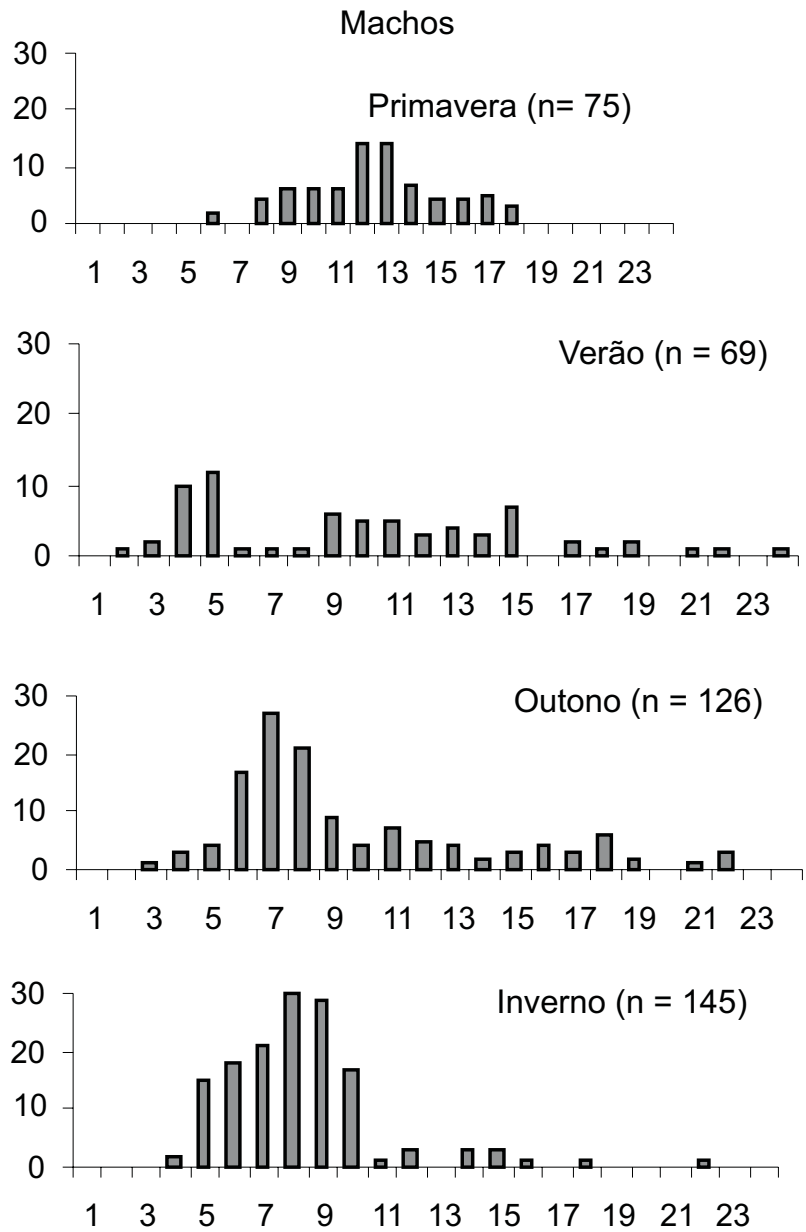

Fêmeas

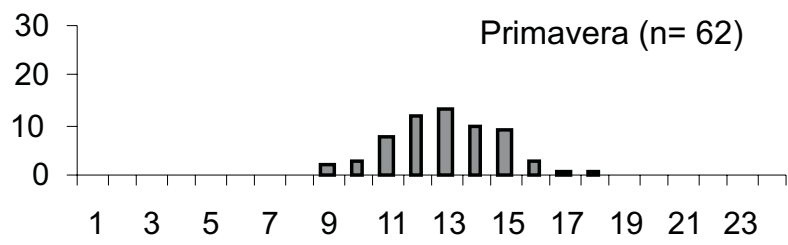

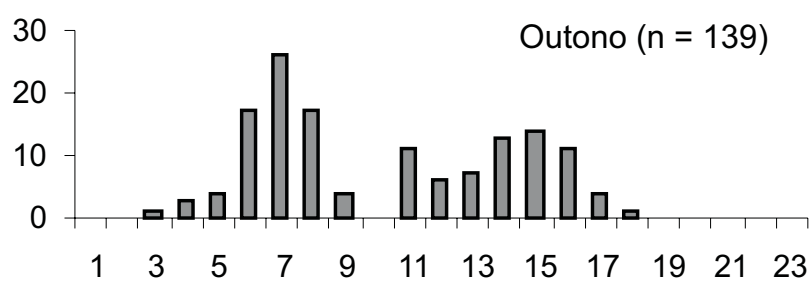

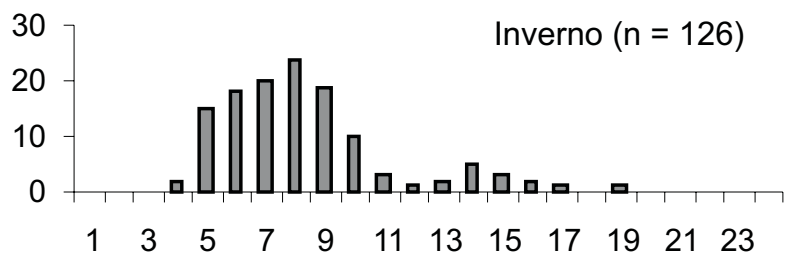

Figura 1. Distribuição das freqüências absolutas do comprimento médio máximo do cefalotórax ( $\mathrm{mm}$ ) de machos e fêmeas de Aegla longirostri coletados no Rio Ibicuí-Mirim, Rio Grande do Sul, mensalmente no período de setembro de 1996 a agosto de 1997. Classes

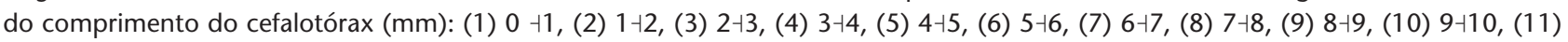
$10 ! 11,(12) 11 \dashv 12,(13) 12 \dashv 13,(14) 13 \dashv 14,(15) 14 \dashv 15,(16) 15 \dashv 16,(17) 16 \dashv 17,(18) 17 \dashv 18,(19) 18 \dashv 19,(20) 19 \dashv 20,(21) 20 \dashv 21,(22)$ $21 \nmid 22,(23) 22 \dashv 23,(24) 23 \dashv 24$.

períodos de recrutamento ao ano para Aegla castro Schmitt, 1942, em abril e dezembro.

As curvas de crescimento em comprimento do cefalotórax, para machos e fêmeas, calculadas por meio da técnica de BERTALANFFY (1938) são descritas pelas seguintes equações: $\mathrm{Ct}=27,9$ $\left[1-\mathrm{e}^{-0,0069(\mathrm{t}+24,78)}\right](\mathrm{n}=415 ; \mathrm{r}=0,77)$ e $\mathrm{Ct}=21,45\left[1-\mathrm{e}^{-0,0048(\mathrm{t}+45,68)}\right]$ ( $\mathrm{n}=391 ; \mathrm{r}=0,83$ ), respectivamente (Figs 2 e 3 ).

$\mathrm{O}$ valor estimado para o comprimento médio máximo do cefalotórax $\left(C_{ұ}\right)$, pela curva de crescimento, foi de $27,9 \mathrm{~mm}$ para machos e $21,45 \mathrm{~mm}$ para fêmeas, sendo estes valores aproximados dos tamanhos dos maiores espécimes amostrados (23,8 $\mathrm{mm}$ para machos e 18,6 $\mathrm{mm}$ para fêmeas).

Os valores do comprimento assintótico não foram semelhantes entre os sexos, mas superiores aos valores encontrados por Bueno et al. (2000) para A. platensis e por Noro \& Buckup (2003) para A. leptodactyla.

Em $A$. longirostri, os machos atingiram tamanhos maiores do que as fêmeas, assim como em outros aeglídeos como $A$. laevis laevis (BAHAMONDE \& LOPEZ 1961), A. odebrechtii (LOPEZ, 1965), A. perobae (Rodrigues \& Hebling 1978), A. platensis (Bueno et al. 2000), A. castro (Swiech-Ayoub \& masunari 2001b) e $A$. leptodactyla (Noro \& Buckup 2003).

O tamanho superior alcançado pelos machos deve-se, provavelmente, ao fato destes direcionarem suas energias principalmente para o crescimento somático, enquanto as fêmeas investem grande parte das energias obtidas para o propósito reprodutivo (maturação das gônadas e produção de ovos) em detrimento do crescimento do corpo e, dessa forma, atingem 

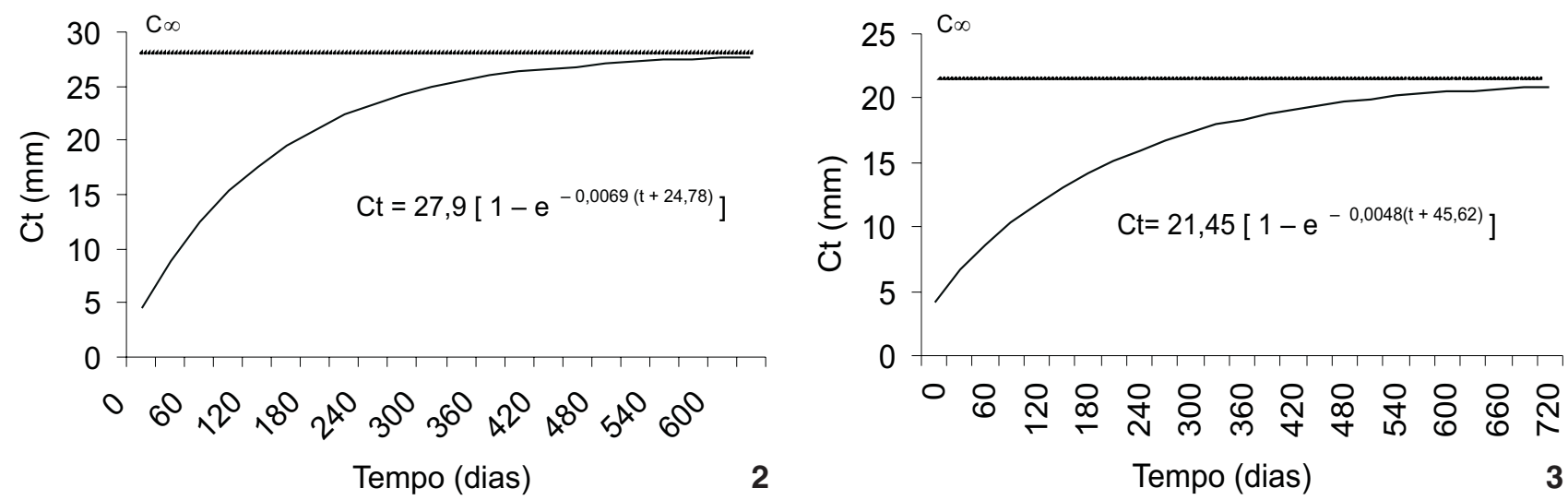

Figuras 2-3. Curvas de crescimento em comprimento do cefalotórax ( $\mathrm{mm}$ ) em machos (2) e fêmeas (3) de Aegla longirostri do Rio lbicuíMirim, Rio Grande do Sul. (Ct) Comprimento do cefalotórax $(\mathrm{mm})$ no tempo t; $\left(\mathrm{C}_{\infty}\right)$ comprimento médio máximo, $(\mathrm{t})$ idade em dias.

tamanhos inferiores aos machos. Ele também pode estar relacionado com o estresse conseqüente da postura dos ovos pelas fêmeas, como foi sugerido por SWIECH-AYoub \& MASUNARI (2001b).

Na maioria dos decápodos, as fêmeas, após a puberdade, apresentam longos períodos de intermuda, principalmente, associadas à postura dos ovos. Como resultado, as fêmeas usualmente sofrem muda com menos freqüência, crescem mais vagarosamente e atingem tamanhos menores que os machos (Hartnoll 1985). Este fato pode explicar, em parte, a taxa de crescimento $(\mathrm{K})$ para os machos de $A$. longirostri ter sido superior $(K=0,0069)$ à das fêmeas $(K=0,0048)$.

Diferentes taxas de mortalidade entre os sexos (Wolf et al. 1975), migração (MonTague 1980), maior facilidade de um dos sexos suportar as adversidades ambientais, desequilíbrio espacial e temporal na utilização dos recursos e padrões comportamentais diferenciados entre os sexos (GIESEL 1972) podem ser alguns dos fatores que, também, influenciam na diferença de tamanho entre machos e fêmeas de uma determinada espécie.

Por meio da curva de crescimento pode-se estimar que $A$. longirostri vive aproximadamente dois anos, tempo semelhante ao obtido para A. platensis estudada por Bueno et al. (2000), A. castro por Swiech-Ayoub \& Masunari (2001b) e A. leptodactyla (2,5 anos) por Noro \& Buckup (2003). Por outro lado, a longevidade de $A$. laevis laevis foi estimada em três anos aproximadamente (BAHAMONDE \& LOPEZ 1961).

As fêmeas de $A$. longirostri apresentaram longevidade um pouco maior (dois anos) em relação aos machos (1,9 ano). Alguns autores relacionam este fato ao comportamento dos machos, que permanecem mais tempo expostos na superfície alimentando-se, disputando e cortejando fêmeas e, dessa forma, eles ficam mais suscetíveis aos predadores e às adversidades ambientais (Christy \& SALMon 1984, Caravello \& Cameron 1987, BACKWELl et al. 2000).

Entretanto, BAHAMONDE \& LOPEZ (1961) observaram maior longevidade para os machos em A. laevis laevis. Segundo hipóteses de Swiech-Ayoub \& Masunari (2001b), este fato pode estar relacionado a uma possível taxa de mortalidade maior entre as fêmeas maturas causada pela predação, pois as massas de ovos as tornam mais pesadas e lentas para a fuga, ou ao estresse sofrido pelas mesmas após o período reprodutivo que as deixa mais vulneráveis.

Com base na comparação das curvas de crescimento linearizadas é possível constatar que não existem diferenças significativas na homogeneidade das variâncias residuais, pois Fc = 0,089 $<\mathrm{F}_{0,05, \mathrm{gl} 4,4}$, sendo que na comparação entre a declividade e a elevação das retas obteve-se um valor de $\mathrm{Fc}>\mathrm{Ft}$, concluindo-se que existe um crescimento diferencial entre os sexos de A. longirostri.

O crescimento em anomuros, apesar das limitadas informações, pode ser considerado como indeterminado, ou seja, o animal sofre ecdises contínuas após a puberdade, porém não significando um crescimento ilimitado (HARTNOLL 1985). Este fato foi verificado na curva de crescimento de A. longirostri, como em outros crustáceos, nos quais o crescimento se torna mais lento à medida que o tamanho do animal aumenta.

A equação de von BERTALANFFY mostrou ser apropriada para o estudo do crescimento de A. longirostri com significância de $95 \%$ e foi escolhida por ser largamente utilizada, especialmente em biologia pesqueira.

Com o presente trabalho foi possível estimar a longevidade de $A$. longirostri, a taxa de crescimento, o tamanho máximo atingido por machos e fêmeas e o crescimento diferencial entre os sexos desta espécie. Desse modo, os dados obtidos neste estudo possibilitaram caracterizar o crescimento de $A$. longirostri fornecendo informações para preservação da espécie.

\section{REFERÊNCIAS BIBLIOGRÁFICAS}

Allen, R.L. 1976. Method for comparing fish growth curves. New Zealand Journal of Marine Freshwater Research, Wellington, 10 (Supl. 4): 687-692.

Backwell, P.R.Y.; J.H. Christy; S.R. Telford; M.D. Jennions, \& N.I. PASSMORE. 2000. Dishonest singling by a fiddler crab. Proceedings of the Royal Society of London, London, 267: 1-6.

Revista Brasileira de Zoologia 23 (2): 408-413, junho 2006 
BAGENAL, T.B. 1978. Methods for assessment of fish production in fresh waters. Oxford, Blackwell Scientific Publications, IBP Handbook no. 3, $3^{\text {rd }}$ ed., 365p.

Bahamonde, N. \& M.T. Lopez. 1961. Estudios biológicos en la populación de Aegla laevis laevis (Latreille) de el Monte (Crustacea, Decapoda, Anomura). Investigaciones Zoológicas Chilenas, Santiago, 7: 19-58.

Barros, M.P. \& N.F. Fontoura. 1996. Crescimento de Potimirim glabra (Kingsley, 1878) (Crustacea, Decapoda, Atyidae) na Praia da Vigia, Garopaba, Santa Catarina, Brasil. Nauplius, Rio Grande, 4: 11-28.

BertalanfFy, L. VON. 1938. A quantitative theory of organic growth (inquiries on growth laws II). Human Biology, Detroit, 10 (1):181-213.

Bond-Buckup, G. \& L. Buckup. 1994. A família Aeglidae (Crustacea, Decapoda, Anomura). Arquivos de Zoologia, São Paulo, 2 (4): $159-346$.

Branco, J.O. \& S. Masunari. 1992. Crescimento de Callinectes danae Smith (Decapoda, Portunidae) da Lagoa da Conceição, Florianópolis, Santa Catarina, Brasil. Revista Brasileira de Zoologia, Curitiba, 9 (1/2): 53-66.

Bueno, A.A.P.; G. Bond-Buckup \& L. Buckup. 2000. Crescimento de Aegla platensis Schmitt em ambiente natural (Crustacea, Decapoda, Aeglidae). Revista Brasileira de Zoologia, Curitiba, 17 (1): 51-60.

Caravello, H.E. \& G.N. Cameron. 1987. The effects of sexual selection on the foraging behavior of the Gulf Coast fiddler crab, Uca panacea. Animal Behavior, Washington, 35 (6): 1864-1874.

CartaXana, A. 2003. Growth of the prawn Palaemon longirostris (Decapoda, Palaemonidae) in Mira River and estuary, SW Portugal. Journal of Crustacean Biology, Laurence, 23 (2): 251-257.

Castiglioni, D.S.; D. Silva-Castiglioni; M.L.N. Fransozo. 2004. Somatic growth of the fiddler crab Uca rapax (Smith, 1870) (Brachyura, Ocypodidae) from two subtropical mangroves. Universidad y Ciencia, Villahermosa, 20 (39): 15-22.

Christy, J.H. \& M. SAlmon. 1984. Ecology and evolution of mating systems of fiddler crabs (Genus Uca). Biological Review, Cambridge, 59: 483-509.

Cracco, E.B. \& N.F. Fontoura. 1996. Dinâmica populacional de Cyrtograpsus angulatus Dana, 1851 no estuário do Rio Tramandaí, Imbé, Rio Grande do Sul, Brasil (Crustacea, Decapoda, Grapsidae). Revista Brasileira de Biologia, Rio de Janeiro, 56 (3) 513-528.

D'incao, F.; M.L. Ruffino; K.G. Silva; A.C. Braga \& L.H.C. MarQUES. 1993. Crescimento de Chasmagnathus granulata Dana, 1851, em um marisma do estuário da Lagoa dos Patos, RS (Decapoda: Grapsidae). Revista Brasileira de Biologia, Rio de Janeiro, 53 (4): 637-643.

FonTOURA, N.F. \& L. Buckup. 1989. O crescimento de Parastacus brasiliensis (Von Marten, 1869) (Crustacea, Decapoda, Parastacidae). Revista Brasileira de Biologia, Rio de Janei- ro, 49 (4): 897: 909.

GIESEL, J.T. 1972. Sex ratio, rate of evolution, and environmental heterogeneity. The American Naturalist, Chicago, 106 (949): 380-387.

Hartnoll, R.G. 1983. Strategies of crustacean growth. Papers from the Conference on the Biology and Evolution of Crustacea. The Australian Museum Memoir, Sydney, 18: 121-131.

HarTNOLL, R.G. 1985. Growth, sexual maturity and reproductive output, p.101-128. In: A.M. WENNER (Ed.) Crustacean Issues: Factors in Adult Growth 3. Rotterdam, A.A. Balkema, 362p.

Lopez, M.T. 1965. Estudios biológicos en Aegla odebrechtti paulensis, Schmitt (Crustacea, Decapoda, Anomura). Boletim de Zoologia da Faculdade de Filosofia, Ciências e Letras, São Paulo, 25: 301-314.

Markus, R. 1971. Elementos de estatística aplicada da Faculdade de Agronomia e Veterinária da UFRGS. Centro Acadêmico Leopoldo Cortez, Porto Alegre, 329p.

Montague, C.L. 1980. A natural history of temperate Western Atlantic fiddler crabs (Genus Uca) with reference to their impact on the salt marsh. Contributions of Marine Science, Bangor, 23: 25-55.

Noro, C.K. \& L. Buckup. 2003. O crescimento de Aegla leptodactyla Buckup \& Rossi (Crustacea, Anomura, Aeglidae). Revista Brasileira de Zoologia, Curitiba, 20 (2): 191-198.

Он, C.-W.; H.-L. SuH; K.-Y. Park; C.-W. MA \& H.-S. Lim. 2002. Growth and reproductive biology of the freshwater shrimp Exopalaemon modestus (Decapoda: Palaemonidae) in a lake of Korea. Journal of Crustacean Biology, Laurence, 22 (2): 357-366.

PAuly, D. 1980. A selection of simple methods for the assessment of tropical fish stocks. FAO Fisheries Circular, Rome, (729): 1-54.

Rodrigues, W. \& N.J. Hebling. 1978. Estudos biológicos em Aegla perobae Hebling \& Rodrigues, 1977 (Decapoda, Anomura). Revista Brasileira de Biologia, Rio de Janeiro, 38 (2): 383390.

SANTOS, E.P. 1978. Dinâmica de Populações Aplicada à Pesca e Piscicultura. São Paulo, Ed. da Universidade de São Paulo, $129 \mathrm{p}$.

Snedecor, C.L. \& W.G. Cochran. 1967. Statistical Method. Ames, The Iowa State University Press, $6^{\text {th }}$ ed., 593p.

SouzA, G.D. \& N.F. Fontoura. 1995. Crescimento de Macrobrachium potiuna no Arroio Sapucaia, município de Gravataí, (Crustacea, Decapoda, Palaemonidae). Revista Brasileira de Biologia, Rio de Janeiro, 55 (Supl. 1): 51-63.

Spiegel, M.R. 1979. Estatística. São Paulo, McGraw-Hill, 580p. Swiech-Ayoub, B.P. \& Masunari, S. 2001a. Biologia reprodutiva de Aegla castro Schmitt (Crustacea, Anomura, Aeglidae) no Buraco do Padre, Ponta Grossa, Paraná, Brasil. Revista Brasileira Zoologia, Curitiba, 18 (3): 1019-1030.

Swiech-Ayoub, B.P. \& S. Masunari. 2001b. Flutuação temporal e espacial de abundância e composição de comprimento da 
carapaça de Aegla castro Schmitt, 1942 (Crustacea, Anomura, Aeglidae) no Buraco do Padre, Ponta Grossa, Paraná, Brasil. Revista Brasileira Zoologia, Curitiba, 18 (3): 1003-1017.

ValentI, W.C.; J.T.C. Mello \& V.L. Lobão. 1987. O crescimento de Macrobrachium acanthurus (WIEGMANN, 1836) do Rio Ribeira de Iguape (Crustacea, Decapoda, Palaemonidae). Revista Brasileira de Biologia, Rio de Janeiro, 47 (3): 349-355.

Valenti, W.C.; J.T.C. Mello \& N. Castagnolli. 1993. Efeito da densidade populacional sobre as curvas de crescimento de Macrobrachium rosembergii (de Man) em cultivo semi-inten- sivo (Crustacea, Palaemoniade). Revista Brasileira de Zoologia, Curitiba, 10 (3): 371-558.

VAlentI, W.C.; J.T.C. Mello \& V.L. Lobão. 1994. Maturation and growth curves of Macrobrachium carcinus (Linnaeus) (Crustacea, Decapoda, Palaemonidae) from Ribeira de Iguape River, southern Brazil. Revista Brasileira de Zoologia, Curitiba, 11 (4): 649-658.

Wolf, P.; S.F. Shanholtaer \& R.J. Reimold. 1975. Population estimates for Uca pugnax on Duplin estuary marsh, Georgia, USA. Crustaceana, Leiden, 29: 79-91.

Recebido em 12.VIII.2005; aceito em 02.V.2006. 